\title{
INCREMENTAL IDENTIFICATION OF TRANSPORT COEFFICIENTS IN CONVECTION-DIFFUSION SYSTEMS
}

\author{
MAKA KARALASHVILI*, SVEN GROß ${ }^{\dagger}$, ADEL MHAMDI*, ARNOLD REUSKEN ${ }^{\dagger}$, AND \\ WOLFGANG MARQUARDT*
}

\begin{abstract}
In this paper, an incremental approach for the identification of a model for transport coefficients in convection-diffusion systems on the basis of high-resolution measurement data is presented. The transport is represented by a convection term with known convective velocity and by a diffusion term with an unknown, generally state-dependent transport coefficient. The identification of the transport model for this transport coefficient constitutes an ill-posed nonlinear inverse problem. We present a novel decomposition approach in which this inverse problem is split into a sequence of inverse subproblems. In the first identification step of this incremental approach a source is estimated by solving an affine-linear inverse problem by means of the conjugate gradient method. In the second identification step a nonlinear inverse problem has to be solved in order to reconstruct a transport coefficient. A Newton-type method using the conjugate gradient method in its inner iteration is used to solve this nonlinear inverse problem of coefficient estimation. Finally, in the third identification step a transport model structure is proposed and identified on the basis of the model-free transport coefficient reconstructed in the two previous steps. The ill-posedness of each inverse problem is examined by using artificially perturbed transient simulation data and appropriate regularization techniques. The identification methodology is illustrated for a three-dimensional convection-diffusion equation which has its origin in the modeling and simulation of energy transport in a laminar wavy film flow.
\end{abstract}

Key words. Modeling, identification, transport, convection-diffusion equation, inverse problem, regularization, parameter estimation.

AMS subject classifications. 15A15, 15A09, 15A23

1. Introduction. Let $\Omega \subset \mathbb{R}^{3}$ be a computational domain, with boundary parts $\partial \Omega=\Gamma_{D} \cup \Gamma_{N}$, where the indices $D$ and $N$ indicate the Dirichlet and Neumann parts of the boundary, respectively. We consider the convection-diffusion equation

$$
\frac{\partial \rho u}{\partial t}+\nabla \cdot(\rho u \mathbf{w})-\nabla \cdot(a \nabla u)=0 \quad \text { in } \Omega \times\left(t_{0}, t_{f}\right],
$$

with initial and boundary conditions

$$
\begin{aligned}
u\left(\mathbf{x}, t_{0}\right) & =u_{0}(\mathbf{x}), \quad \mathbf{x} \in \Omega, \\
u(\mathbf{x}, t) & =g_{D}(\mathbf{x}, t), \quad(\mathbf{x}, t) \in \Gamma_{D} \times\left[t_{0}, t_{f}\right], \\
\frac{\partial u}{\partial n}(\mathbf{x}, t) & =g_{N}(\mathbf{x}, t), \quad(\mathbf{x}, t) \in \Gamma_{N} \times\left[t_{0}, t_{f}\right] .
\end{aligned}
$$

The scalar state variable $u(\mathbf{x}, t)$ represents, e.g. specific enthalpy in case of energy transport or mass density in case of mass transport. $\rho(\mathbf{x}, t)$ stands for the density of the fluid. The vector field $\mathbf{w}(\mathbf{x}, t) \in \mathbb{R}^{3}$ represents velocity and is assumed to be known. The scalar function $a(\cdot)$ denotes the unknown, in general state-dependent, transport coefficient.

The transport coefficient describes complicated transport phenomena, for which a multitude of competing candidate model structures can be formulated on the basis

*AVT-Process Systems Engineering, RWTH Aachen University, Turmstrasse 46, D-52064 Aachen, Germany (\{maka.karalashvili, adel.mhamdi, wolfgang.marquardt\}@avt.rwth-aachen.de).

$\dagger$ Numerical Mathematics, RWTH Aachen University, Templergraben 55, D-52056 Aachen, Germany (\{gross, reusken\}@igpm.rwth-aachen.de). 
of different assumptions and theories. Experimental data should be used to estimate parameters that occur in these candidate models and to discriminate between the competing candidate models using some reasonable measure of model validity.

The identification of transport coefficients from appropriate measurement data, such as temperature or concentration, belongs to the class of ill-posed inverse problems. Many studies on the estimation of transport coefficients are available. A well-established technique for the identification of transport coefficients, as a function of states and time, relies on an optimization-based formulation which is used in the framework of a coefficient inverse problem, cf. [2, 14]. In this approach, the reconstruction of the transport coefficient in model (1.1) uses suitable transient measurement data $u_{m}(\mathbf{x}, t),(\mathbf{x}, t) \in \Omega \times\left[t_{0}, t_{f}\right]$. It is often assumed that the initial and boundary conditions of the problem are known. Quite some literature is available on the subject (cf. $[3,11,28]$ and the references therein); the treatment, however, is typically restricted to one or two space dimensions. Furthermore, these studies do not aim at the reconstruction of a suitable transport model (structure and parameters) for transport coefficients.

In the so-called simultaneous approach, problem (1.1) for the identification of a model (structure and parameters) for the transport coefficient is solved for each model candidate. This leads to a large number of complex estimation problems. As a consequence, the discrimination between competing transport model candidates requires high computational effort. Furthermore, if a model candidate for the transport coefficient contains uncertainty or structural errors, this approach often yields biased or poor estimates [29]. Often satisfactory results can only be achieved if the correct model structure for the transport coefficient is known. In the present work, in contrast, we use a fundamentally different, so-called incremental approach [22] for the identification of a structured model for the transport coefficient.

In the incremental identification approach, incremental modeling interplays with the incremental identification. In incremental modeling, the structure of a model to be identified is refined step by step by specifying submodels gradually in a sequence of successive refinement levels. Consequently, more transparency concerning the individual decisions during the modeling process can be gained. The incremental identification of a model reflects the steps of incremental modeling straightforwardly by splitting up the identification problem into a sequence of subproblems. The discrimination between the candidate models turns out to become more flexible as the replacement of a submodel on a certain model refinement level affects only the submodels on the following levels. Often this also leads to benefits in terms of less computational effort. The incremental strategy already proved to be an efficient and robust alternative for the mechanistic modeling of kinetic phenomena in multi-phase systems [23], the reconstruction of diffusion coefficients in liquids [6] and the identification of complex reaction kinetics in homogeneous systems [9].

In this paper, we present and investigate the incremental method of modeling and identification for the class of inverse transport coefficient problems described by eq. (1.1). The application of the incremental approach to this class of problems is new. As a first step in the analysis of this technique we show, based on simulated data assuming a time- and space-dependent transport coefficient, that it can be reconstructed without using any a-priori knowledge on its functional representation. We assume, however, that the model structure for the parametric model of this transport coefficient is known. Nevertheless, the incremental identification technique can be directly applied for the case when the model structure is unknown and has to be determined 
from the data and prior knowledge on candidate model structures. Furthermore, in case of different model candidates for the transport coefficient, the additional procedure of model discrimination does not affect the overall technique described in this paper. We analyse this identification approach and show that the method yields satisfactory results if we add noise to the data. These results indicate that the incremental approach is a promising method for this class of transport identification problems.

The paper is organized as follows. The incremental approach of modeling and identification of transport phenomena is presented in Section 2. The optimizationbased formulations for the inverse problems arising in the three steps of the identification procedure are given in Section 3. We also shortly describe the solution strategies used to solve the inverse problems arising in the first two steps of the incremental identification procedure. In Section 4 we present results of extensive numerical experiments for the identification of a model for the transport coefficient in a three-dimensional convection-diffusion problem of type (1.1). This model problem is motivated by research on energy transport in wavy films, using effective transport coefficients $[8,12,30]$. Section 5 contains some conclusions and remarks concerning future work.

2. Incremental modeling and identification. The key idea of the incremental approach is the gradual refinement of the model structure during identification, reflecting the incremental steps which are common in model development. The main steps of model development and their relation to incremental model identification are outlined in the following.

2.1. Incremental modeling. Incremental modeling aims at a generic and structured process for the development of model equations [22, 23]. The starting point is the formulation of the balance equations. The balance equation for a scalar state $u(\mathbf{x}, t)$, that denotes the specific quantity conserved, is given by

$$
\frac{\partial \rho u}{\partial t}+\nabla \cdot \mathbf{j}=0
$$

Here, $\mathbf{j}$ is the flux vector, that governs the rate of transfer of the conserved physical quantity. This vector consists of a convective and diffusive part:

$$
\mathbf{j}=\rho u \mathbf{w}+\mathbf{q} .
$$

The use of the continuity equation leads to the convection-diffusion equation

$$
\text { model } B: \quad \frac{\partial u}{\partial t}+\mathbf{w} \cdot \nabla u=-\frac{1}{\rho} \nabla \cdot \mathbf{q} \quad \text { in } \Omega \times\left(t_{0}, t_{f}\right] .
$$

We do not refine this equation any further, i. e. at this decision level no additional assumptions are made about the potentially uncertain constitutive relation for the diffusive flux vector $\mathbf{q}$.

In the next step, the model is refined by specifying a functional form of the flux q. Often a constitutive relation is used, for example, Fourier's law in heat transfer or Fick's law in mass transfer, which can be cast as

$$
\text { model } F: \quad \mathbf{q}=-a \nabla u \quad \text { in } \Omega \times\left(t_{0}, t_{f}\right],
$$

with an unknown transport coefficient $a$. In empirical approaches one usually distinguishes different transport mechanisms, namely transport by turbulent or molecular 
mechanisms, with or without convection [10]. Accordingly, the transport coefficient in (2.2) is represented as a sum of two contributions - the known molecular part $a_{\mathrm{mol}}$, the molecular transport coefficient corresponding to molecular transport (e.g. heat conduction through the fluid) and the unknown remaining part $a_{w}(\mathbf{x}, t)$ capturing the remaining transport effects (e.g. due to turbulence or other transport enhancing effects). In the following, we call $a_{w}(\mathbf{x}, t)$ the enhanced transport coefficient. Thus,

$$
a(\mathbf{x}, t)=a_{\mathrm{mol}}+a_{w}(\mathbf{x}, t), \quad(\mathbf{x}, t) \in \Omega \times\left[t_{0}, t_{f}\right] .
$$

In the final step of the incremental modeling procedure, a further refinement level is added by specifying a constitutive relation for the enhanced transport coefficient to close the model. We formulate it in a generic way,

$$
\text { model } T: \quad a_{w}(\mathbf{x}, t)=f_{\mathrm{w}}(u(\mathbf{x}, t), \mathbf{x}, t, \theta),
$$

to correlate $a_{w}$ with the state $u$ and model parameters $\theta \in \mathbb{R}^{n}$.

2.2. Incremental identification. The incremental identification directly follows the steps of model development [23]. We assume throughout, that appropriate transient measurement data at sufficiently high resolution in space $\mathbf{x}$ and time $t$ are available. A schematic picture of the procedure is given in Fig. 1.

We rewrite the balance equation (2.1) as

$$
\frac{\partial u}{\partial t}+\mathbf{w} \cdot \nabla u=F \quad \text { in } \Omega \times\left(t_{0}, t_{f}\right]
$$

with

$$
F(\mathbf{x}, t)=-\nabla \cdot \mathbf{q}(\mathbf{x}, t), \quad(\mathbf{x}, t) \in \Omega \times\left(t_{0}, t_{f}\right] .
$$

Here, we have assumed for simplicity a constant density normalized to $\rho=1$. In the first step of the incremental identification procedure, the (artificial) source $F(\mathbf{x}, t)$ is estimated, as a function of space and time, from the balance equation (2.5) with proper initial and boundary conditions, on the basis of suitable measurements $u_{m}(\mathbf{x}, t)$ of the state $u(\mathbf{x}, t)$. This is a typical example of a source inverse problem [2].

The incremental identification at the next level uses the estimated source $F(\mathbf{x}, t)$ as model-based measurement data together with the transient measurements $u_{m}(\mathbf{x}, t)$ to reconstruct the transport coefficient $a_{w}(\mathbf{x}, t)$. Hence, accounting $(2.3),(2.2)$ and $(2.6), a_{w}(\mathbf{x}, t)$ is to be estimated from the equation

$$
-\nabla \cdot\left(\left(a_{\mathrm{mol}}+a_{w}\right) \nabla u\right)=-F \quad \text { in } \Omega \times\left(t_{0}, t_{f}\right],
$$

which corresponds to a coefficient inverse problem [2].

In the third step of the identification procedure the reconstructed coefficient $a_{w}(\mathbf{x}, t)$ is correlated with states as in (2.4) by solving a parameter estimation problem. Different model candidates involving the state $u$ and model parameters $\theta$ can be considered here. The measurement data are used to estimate parameters for each candidate model. The best model is selected by carrying out a model discrimination between candidates using some measure of model validity [29].

In this paper, we focus on the inverse problems that arise in the first two steps of the incremental identification approach. In the third identification step we estimate model parameters in a model structure for the transport coefficient which is assumed 


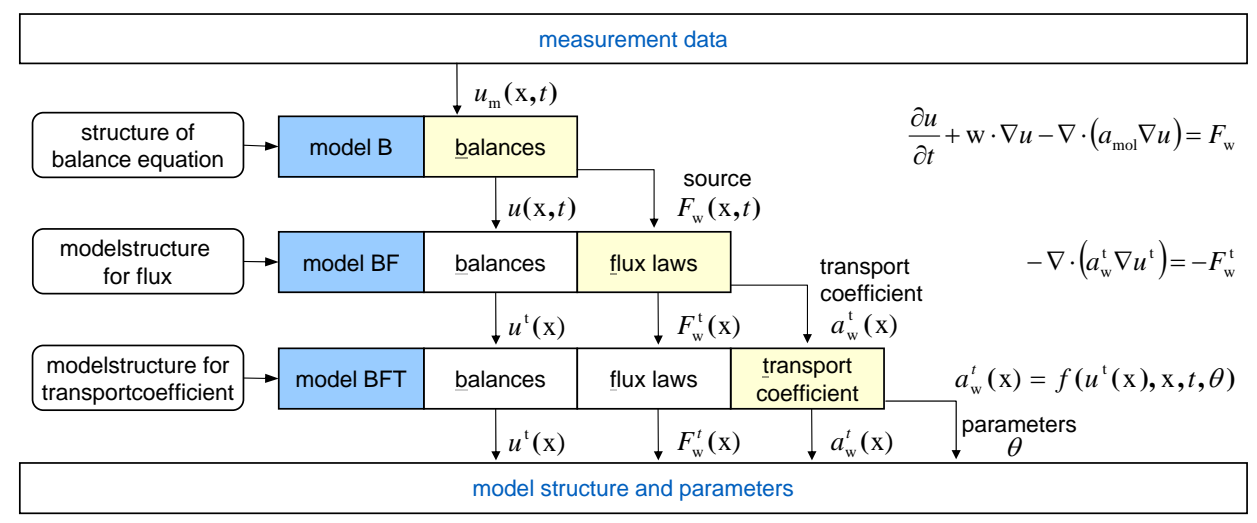

FIG. 1. Incremental modeling and identification of transport phenomena.

to be known, thus restricting ourselves to the estimation of one given model for the transport coefficient.

For the numerical treatment of the source inverse problem in the first step it is very convenient to consider a variant of (2.5) which uses the expression (2.7) for the transport coefficient. This leads to

$$
F(\mathbf{x}, t)=\nabla \cdot\left(a_{\mathrm{mol}} \nabla u(\mathbf{x}, t)\right)+F_{w}(\mathbf{x}, t), \quad(\mathbf{x}, t) \in \Omega \times\left(t_{0}, t_{f}\right] .
$$

As a result, instead of $F(\mathbf{x}, t)$ it suffices to estimate the enhanced part $F_{w}(\mathbf{x}, t)$ of the source term on the basis of transient measurement data $u_{m}(\mathbf{x}, t)$ (cf. Fig. 1). Consequently, in the first step of the identification procedure one has to reconstruct the source term $F_{w}(\mathbf{x}, t)$ in the following convection-diffusion equation

$$
\operatorname{model} \bar{B}: \quad \frac{\partial u}{\partial t}+\mathbf{w} \cdot \nabla u-\nabla \cdot\left(a_{\mathrm{mol}} \nabla u\right)=F_{w} \quad \text { in } \Omega \times\left(t_{0}, t_{f}\right],
$$

with initial and boundary conditions

$$
\begin{aligned}
u\left(\mathbf{x}, t_{0}\right) & =u_{0}(\mathbf{x}), \mathbf{x} \in \Omega, \\
u(\mathbf{x}, t) & =g_{D}(\mathbf{x}, t),(\mathbf{x}, t) \in \Gamma_{D} \times\left[t_{0}, t_{f}\right], \\
\frac{\partial u}{\partial n}(\mathbf{x}, t) & =g_{N}(\mathbf{x}, t),(\mathbf{x}, t) \in \Gamma_{N} \times\left[t_{0}, t_{f}\right] .
\end{aligned}
$$

Compared to (2.5), we now have a convection-diffusion problem instead of a pure convection problem. Due to the diffusion part, the numerical treatment becomes easier. Furthermore, for $u$ we can now use the same boundary conditions as in (1.1b).

In the second step of the incremental identification procedure, one has to determine the coefficient $a_{w}(\mathbf{x}, t)$ in the diffusion equation (cf. Fig. 1)

$$
\text { model } \bar{F}: \quad-\nabla \cdot\left(a_{w}^{t} \nabla u^{t}\right)=-F_{w}^{t} \quad \text { in } \Omega,
$$

with boundary conditions

$$
\begin{aligned}
u^{t}(\mathbf{x}) & =g_{D}^{t}(\mathbf{x}), & \mathbf{x} & \in \Gamma_{D}, \\
\frac{\partial u^{t}}{\partial n}(\mathbf{x}) & =g_{N}^{t}(\mathbf{x}), & \mathbf{x} & \in \Gamma_{N} .
\end{aligned}
$$


Here, for a space and time dependent function $\xi(\mathbf{x}, t)$ we have introduced the notation $\xi^{t}(\mathbf{x}):=\xi(\mathbf{x}, t),(\mathbf{x}, t) \in \Omega \times\left[t_{0}, t_{f}\right]$ to decouple the function values in time instants. In $(2.10)$ we thus have a steady-state diffusion problem for each given $t \in\left[t_{0}, t_{f}\right]$.

In the third step of the identification procedure, the reconstructed coefficients $a_{w}^{t}(\mathbf{x})$ at selected times $t \in\left[t_{0}, t_{f}\right]$ are correlated with states $u^{t}(\mathbf{x})$ and parameters $\theta$ in the parametric model (cf. Fig. 1)

$$
\text { model } \bar{T}: \quad a_{w}^{t}(\mathbf{x})=f_{\mathrm{w}}\left(u^{t}(\mathbf{x}), \mathbf{x}, t, \theta\right), \quad \theta \in \mathbb{R}^{n} .
$$

We briefly compare the incremental identification approach to the established simultaneous identification approach. For this purpose, we insert the relation (2.4) into the flux model and insert the result into the convection-diffusion equation (1.1a),

$$
\begin{array}{r}
\operatorname{model} \overline{B F T}: \quad \frac{\partial u}{\partial t}+\mathbf{w} \cdot \nabla u-\nabla \cdot(f(u(\mathbf{x}, t), \mathbf{x}, t, \theta) \nabla u)=0 \\
\text { in } \Omega \times\left(t_{0}, t_{f}\right],
\end{array}
$$

with initial and boundary conditions

$$
\begin{aligned}
u\left(\mathbf{x}, t_{0}\right) & =u_{0}(\mathbf{x}), \quad \mathbf{x} \in \Omega, \\
u(\mathbf{x}, t) & =g_{D}(\mathbf{x}, t), \quad(\mathbf{x}, t) \in \Gamma_{D} \times\left[t_{0}, t_{f}\right], \\
\frac{\partial u}{\partial n}(\mathbf{x}, t) & =g_{N}(\mathbf{x}, t), \quad(\mathbf{x}, t) \in \Gamma_{N} \times\left[t_{0}, t_{f}\right] .
\end{aligned}
$$

While the incremental approach decomposes the identification process for the transport coefficient in three steps, in the simultaneous approach the models for the flux (e.g. (2.2)) and for the transport coefficient (e.g. (2.4)) are collected in one equation (2.12b). Hence, all the assumptions made during the modeling will simultaneously influence the identification. Due to this, the level of uncertainty of the simultaneous problem (2.12) has increased, leading to a higher risk of poor estimates.

A further advantage of the incremental approach is that, for known velocity $\mathbf{w}(\mathbf{x}, t)$ and molecular transport coefficient $a_{\mathrm{mol}}$, it suffices to reconstruct the source $F_{w}(\mathbf{x}, t)$ at the first level and the enhanced transport coefficients $a_{w}^{t}(\mathbf{x})$ at the second level only once. The complexity due to the elimination of candidate models for the transport coefficient affects the third (final) level only, thus allowing for a more systematic derivation of suitable candidate models.

Compared to the simultaneous problem (2.12), where a nonlinear coefficient inverse problem in space and time has to be solved, the incremental identification procedure has advantages from the optimization point of view. The reconstruction of the source in the first step results in a dynamic optimization problem, which is affinelinear in the unknown. The latter property implies that (compared to a strongly nonlinear case) relatively simple and efficient optimization methods can be applied. In the second step of the identification, we have to deal with a nonlinear coefficient inverse problem which, however, is of steady-state type for each given time $t$, cf. (2.10). In this sense the incremental approach decouples dynamics and nonlinearity, which has advantages for the numerical treatment of nonlinear inverse problems for evolution equations in three dimensions. Furthermore, the-in the worst case-combinatorial problem of identifying a suitable model structure is decoupled from the problem of inversion of differential equations.

The estimation problems arising in the first two steps of the incremental approach are typical inverse problems, ill-posed by nature. This raises, however, the question 
of error propagation through the sequence of inverse problems. This issue is studied for the illustrative model problem in Section 4.

3. Formulation and solution of the inverse problems. The inverse problems resulting in the three incremental steps are formulated as optimization problems and solved with state-of-the-art methods $[1,2,14]$.

3.1. Problem formulations. In the first step of the incremental identification procedure, the source $F_{w}$ should minimize the quadratic objective functional

$$
J_{1}\left(F_{w}\right)=\frac{1}{2} \int_{t_{0}}^{t_{f}} \int_{\Omega}\left[u\left(\mathbf{x}, t ; F_{w}\right)-u_{m}(\mathbf{x}, t)\right]^{2} d \mathbf{x} d t
$$

with suitable transient measurement data $u_{m}(\mathbf{x}, t),(\mathbf{x}, t) \in \Omega \times\left[t_{0}, t_{f}\right]$. Here $u\left(\mathbf{x}, t ; F_{w}\right)$ is the solution of the direct problem (2.9) with known initial and boundary conditions $u_{0}, g_{D}$ and $g_{N}$, respectively.

Similarly, the second identification step concerns the estimation of the enhanced transport coefficients $a_{w}^{t}(\mathbf{x})$ as a functions of space $\mathbf{x}$ at selected times $t \in\left[t_{0}, t_{f}\right]$, using the previously estimated source $F_{w}^{t}(\mathbf{x})$ and the measurement data $u_{m}^{t}(\mathbf{x})$. The optimization-based formulation of this coefficient inverse problem consists of the minimization of the objective functional

$$
J_{2}\left(a_{w}^{t}\right)=\frac{1}{2} \int_{\Omega}\left[u^{t}\left(\mathbf{x} ; a_{w}^{t}\right)-u_{m}^{t}(\mathbf{x})\right]^{2} d \mathbf{x} .
$$

Here $u^{t}\left(\mathbf{x} ; a_{w}^{t}\right)$ denotes the solution of the direct problem (2.10) for given $a_{w}^{t}$.

Finally, in the third identification step, a standard least-squares problem is posed such, that the model $f\left(u^{t}(\mathbf{x}), \mathbf{x}, t, \theta\right)$ with parameters $\theta \in \mathbb{R}^{n}$ (cf. (2.11)) minimizes the objective functional

$$
J_{3}\left(f\left(u^{t}(\mathbf{x}), \mathbf{x}, t, \theta\right)\right)=\frac{1}{2} \sum_{t} \int_{\Omega}\left[a_{w}^{t}(\mathbf{x})-f\left(u^{t}(\mathbf{x}), \mathbf{x}, t, \theta\right)\right]^{2} d \mathbf{x}
$$

where $a_{w}^{t}(\mathbf{x})$ represents the reconstructed transport coefficient at times $t \in\left[t_{0}, t_{f}\right]$. This estimation problem depends strongly on the availability of candidate models $f(\cdot)$. In cases where no reasonable (structured) model can be formulated (i.e. the model structure is unknown), a general parameterization capable of approximating functions from a sufficiently large class should be introduced for the transport coefficients $a_{w}^{t}$ and the model parameters $\theta$ should be estimated by means of data-driven techniques [20]. In case of (structured) available model candidates, e.g. from physical considerations and/or a priori knowledge, the parameters $\theta$ are to be estimated for each candidate model. Subsequently, the adequacy of the different candidates has to be quantified with the use of model discrimination approaches in order to choose the best model for the transport coefficient [29].

3.2. Numerical optimization strategies. For the solution of the optimization problem in the first step of the incremental approach, the conjugate gradient method is used $[15,24]$. The optimization problem in the second step is solved by means of an inexact Newton-type method, which is an appropriate technique for a large class of nonlinear inverse problems [14]. In this paper, regularization is only introduced via the fixed spatial and temporal discretization and by means of a suitable stopping criterion for the optimization algorithms. Hence, for a given discretization, the number 
of optimization iterations serves as the only regularization parameter [14]. Either the heuristic L-curve method [19] or the discrepancy principle [14] is used to determine an appropriate value of this parameter.

The realisation of the two optimization strategies used for the solution of the inverse problems within the first two steps of the incremental identification procedure, require two nontrivial values: the direction of descent of the objective functional and the length of descent along that direction. In the following subsections we will infer these issues in brief. For the derivation of the arising adjoint and sensitivity problems therein refer to e.g. $[1,2]$.

Finally, for the solution of an unconstrained minimization problem (3.3) in the third step of the incremental identification procedure, we use standard solution techniques for least-squares problems [24]. In our case study (cf. Section 4), we only consider a single model candidate for the transport coefficient, hence implicitly assuming that the model structure is known.

3.2.1. Estimation of the source $F_{w}(\mathbf{x}, t)$. For the minimization of the objective functional (3.1) with constraints (2.9), the conjugate gradient (CG) method is used $[1,15,17,24]$. Here, the unknown function is sequentially updated during the iteration process starting from some initial guess by moving along a (conjugate) descent direction $\tilde{F}_{w}^{n}$ with an optimal step length $\mu^{n}$ at optimization iteration $n$ with

$$
F_{w}^{n+1}=F_{w}^{n}-\mu^{n} \tilde{F}_{w}^{n} .
$$

Each new descent direction $\tilde{F}_{w}^{n}$ in (3.4) is calculated according to

$$
\tilde{F}_{w}^{n}=\nabla J_{1}\left(F_{w}^{n}\right)+\gamma^{n} \tilde{F}_{w}^{n-1},
$$

with $\nabla J_{1}\left(F_{w}^{n}\right)$ the gradient of the objective functional (3.1) and $\gamma^{n}$ the conjugate coefficient. It can be shown (cf. e.g. [1]) that this gradient satisfies

$$
\nabla J_{1}\left(F_{w}^{n}\right)=\varphi_{1} \quad \text { in } \Omega \times\left[t_{0}, t_{f}\right],
$$

where the adjoint variable $\varphi_{1}$ is the solution of the adjoint problem

$$
\begin{aligned}
-\frac{\partial \varphi_{1}}{\partial t}-\mathbf{w} \cdot \nabla \varphi_{1}-a_{\mathrm{mol}} \Delta \varphi_{1} & =\left[u\left(F_{w}^{n}\right)-u_{m}\right] \quad \text { in } \Omega \times\left[t_{0}, t_{f}\right), \\
\varphi_{1}\left(\mathbf{x}, t_{f}\right) & =0, \quad \mathbf{x} \in \Omega, \\
\varphi_{1}(\mathbf{x}, t) & =0, \quad(\mathbf{x}, t) \in \Gamma_{D} \times\left[t_{0}, t_{f}\right], \\
\frac{\partial \varphi_{1}}{\partial n}(\mathbf{x}, t) & =0, \quad(\mathbf{x}, t) \in \Gamma_{N} \times\left[t_{0}, t_{f}\right],
\end{aligned}
$$

with $u\left(F_{w}^{n}\right)$ the solution of the direct problem (2.9). In contrast to this direct problem, we now have a condition at final time $t_{f}$. Going backwards in time (by introducing a new time variable $t_{f}-t$ ), equation (3.7) shows exactly the same structure as the direct problem (2.9), only with different initial and boundary conditions.

The step length $\mu^{n}$ in (3.4) is obtained by solving a one-dimensional minimization problem resulting in

$$
\mu^{n}=\frac{\left(u_{m}-u\left(F_{w}^{n}\right), S_{1}\left(\tilde{F}_{w}^{n}\right)\right)_{H}}{\left\|S_{1}\left(\tilde{F}_{w}^{n}\right)\right\|_{H}^{2}} .
$$


Here, we assumed $H$ to be a given (Hilbert) space; $S_{1}$ is the solution of the sensitivity problem given by

$$
\begin{array}{rlrl}
\frac{\partial S_{1}}{\partial t}+\mathbf{w} \cdot \nabla S_{1}-a_{\mathrm{mol}} \Delta S_{1} & =\tilde{F}_{w}^{n} & & \text { in } \Omega \times\left(t_{0}, t_{f}\right], \\
S_{1}\left(\mathbf{x}, t_{0}\right) & =0, & \mathbf{x} \in \Omega, \\
S_{1}(\mathbf{x}, t) & =0, & & (\mathbf{x}, t) \in \Gamma_{D} \times\left[t_{0}, t_{f}\right], \\
\frac{\partial S_{1}}{\partial n}(\mathbf{x}, t) & =0, & & (\mathbf{x}, t) \in \Gamma_{N} \times\left[t_{0}, t_{f}\right] .
\end{array}
$$

This partial differential equation has exactly the same structure as the corresponding direct problem (2.9), only the initial and boundary conditions are different.

Thus, the CG algorithm for minimizing $J_{1}$ requires the solution of three very similar problems in every iteration, namely the direct, the adjoint and the sensitivity problem. However, due to the linearity of the involved equations only two problems - the adjoint (3.7) and the sensitivity (3.9) problem - have to be solved [17].

3.2.2. Estimation of the transport coefficients $a_{w}^{t}(\mathbf{x})$. For the minimization of the objective functional (3.2) with constraints (2.10) a Newton-type method is used. The basic idea of the truncated Newton-CGNE method is the computation of a regularized approximation of the linearized problem by an inner iteration, namely a CG method [18]. The termination criterion used for the CG (inner) iteration relates this method to the general class of inexact Newton methods $[18,24]$. The sequential update formula for the unknown functions $a_{w}^{t}$ at selected times $t \in\left[t_{0}, t_{f}\right]$ during the Newton (outer) iterations $k=1,2, \ldots$ is given by

$$
a_{w}^{t, k+1}=a_{w}^{t, k}+x^{n_{\star}}
$$

with the update $x^{n_{\star}}$, the result of the $n_{\star}$-th CG iteration. In analogy to (3.4), the descent direction and step length are required for the sequential update of this quantity within each CG iteration.

The descent direction $\tilde{a}_{w}^{t, n}$ at given time $t$ and CG iteration $n$, and the step length $\alpha^{n}$ are obtained by the similar formulas as in (3.5),(3.8), respectively [18]. The only difference consists in replacing $\nabla J_{1}\left(F_{w}^{n}\right)$ in (3.5) by the adjoint variable $\varphi_{2}^{t}$ - the solution of the following adjoint problem

$$
\begin{aligned}
-\nabla \cdot\left(a_{w}^{t, n} \nabla \varphi_{2}^{t}\right) & =\left[u^{t}\left(\mathbf{x} ; a_{w}^{t, n}\right)-u_{m}^{t}(\mathbf{x})\right] \quad \text { in } \Omega, \\
\varphi_{2}^{t}(\mathbf{x}) & =0, \quad \mathbf{x} \in \Gamma_{D}, \\
\frac{\partial \varphi_{2}^{t}}{\partial n}(\mathbf{x}) & =0, \quad \mathbf{x} \in \Gamma_{N} .
\end{aligned}
$$

Here, $u^{t}\left(\mathbf{x} ; a_{w}^{t, n}\right)$ denotes the solution of the corresponding direct problem (2.10) for a given time $t$ and value of $a_{w}^{t, n}(\mathbf{x})$. This problem has the same structure as the direct problem (2.10).

The step length $\alpha^{n}$ is calculated by replacing $S_{1}$ in (3.8) with the solution $S_{2}^{t}$ at time $t$ of the sensitivity problem given now as

$$
\begin{aligned}
-\nabla \cdot\left(a_{w}^{t, n} \nabla S_{2}^{t}\right) & =\nabla \cdot\left(\tilde{a}_{w}^{t, n} \nabla u^{t}\right) \quad \text { in } \Omega \\
S_{2}^{t}(\mathbf{x}) & =0, \quad \mathbf{x} \in \Gamma_{D}, \\
\frac{\partial S_{2}^{t}}{\partial n}(\mathbf{x}) & =0, \quad \mathbf{x} \in \Gamma_{N} .
\end{aligned}
$$


This equation has the same structure as the corresponding direct problem. Note, however, that apart from the different boundary conditions one also has a specific right-hand side in this sensitivity equation, which arises due to the nonlinearity of the coefficient inverse problem.

In the truncated Newton-CGNE method, one has to solve an adjoint and a sensitivity problems in each CG iteration for the determination of the descent direction and the step length, respectively. Due to the nonlinearity of the estimation problem, however, the direct problem (2.10) has to be solved in each Newton iteration.

3.2.3. Solution of the underlying PDE problems. All direct, sensitivity and adjoint problems to be solved as part of the numerical optimization strategies described above are either of elliptic or parabolic (convection-diffusion) type. Hence, similar numerical techniques can be employed for their solution.

The solutions of all 3D problems are calculated by means of the software package DROPS [13]. DROPS is based on multi-level nested grids and conforming finite element discretization methods. For time discretization a standard one-step $\theta$-method is used. For the space discretization piecewise linear finite elements on a tetrahedral grid are employed. The resulting discrete systems of linear equations are solved by suitable Krylov subspace methods. In case of the convection-diffusion equations (i.e. (2.9), (3.7), (3.9)) we use a preconditioned Generalized Minimal Residuals (GMRES) method. For the diffusion problems (i.e. (2.10), (3.11), (3.12)) a preconditioned conjugate gradient (PCG) method is applied [27]. For the simulations presented in this paper the SSOR method is used for preconditioning. Other options, for example multigrid solvers, are available in DROPS. In this paper we do not study efficiency of these solvers for the direct, the sensitivity and the adjoint problems. We use a fixed (quasi-uniform) mesh for discretization and prescribe a tolerance with which the discrete linear systems are solved.

4. Illustrative case study. In this section, the incremental approach is illustrated for a problem motivated by the identification of energy transport in laminar wavy film flows. The complex dynamics of the nonlinear surface waves typically present in film flows $[16,25]$ renders a direct transient simulation in 3D numerically very complicated and computationally expensive. Therefore, manageable approximate descriptions, yet accurately modeling the underlying transport processes, have gained increasing importance in the engineering literature to support the design of technical systems [8]. A possible simplified model is as follows. In order to reduce the problem complexity, the 3D time-varying domain $\Omega_{W}$ corresponding to the liquid phase is mapped to a $3 \mathrm{D}$ time-invariant waveless domain $\Omega:=\left(0, L_{x}\right) \times\left(0, L_{y}\right) \times\left(0, L_{z}\right) \subset \mathbb{R}^{3}$. This reduction is compensated by the introduction of a space- and time-dependent effective transport coefficient $a_{\mathrm{eff}}(\mathbf{x}, t)[8,12,30]$ to capture all wave-induced transport effects in this flat film geometry.

Such a flat film model is considered in the remainder. It consists of a convectiondiffusion system which describes energy transport in a single component fluid on the flat (rectangular) domain $\Omega$ with boundary $\Gamma=\partial \Omega$, with parts $\Gamma=\Gamma_{\text {in }} \cup \Gamma_{\text {wall }} \cup$ $\Gamma_{\text {out }} \cup \Gamma_{r}$ defined as

$$
\begin{aligned}
\Gamma_{\text {in }} & =\{(x, y, z) \in \Gamma: x=0\} \subset \Gamma_{D}-\text { the inflow boundary }, \\
\Gamma_{\text {wall }} & =\{(x, y, z) \in \Gamma: y=0\} \subset \Gamma_{D}-\text { the wall boundary } \\
\Gamma_{\text {out }} & =\left\{(x, y, z) \in \Gamma: x=L_{x}\right\} \subset \Gamma_{N}-\text { the outflow boundary } \\
\Gamma_{r} & =\Gamma \backslash\left(\Gamma_{\text {in }} \cup \Gamma_{\text {wall }} \cup \Gamma_{\text {out }}\right) \subset \Gamma_{N}-\text { the remaining boundaries } .
\end{aligned}
$$


The state variable in $(1.1)$ is now $u(\mathbf{x}, t)=c T(\mathbf{x}, t)$ with the temperature $T(\mathbf{x}, t)$ and thermal capacity $c$. Whereas, for the transport coefficient $a(\mathbf{x}, t)=\rho a_{\mathrm{eff}}(\mathbf{x}, t)$ applies, with the effective thermal diffusivity $a_{\mathrm{eff}}(\mathbf{x}, t)$ and density $\rho$. We assume $c$ and $\rho$ to be constants. The unit cube $\Omega=(0,1)^{3}\left[\mathrm{~mm}^{3}\right]$ is considered as computational domain for simplicity of presentation and to avoid possible numerical complications due to anisotropy effects. $x$ corresponds to the flow direction of the falling film and $y$ is the direction along the film thickness. The velocity $\mathbf{w}(\mathbf{x}, t)$ is given by a Nusseltprofile, i.e. $\mathbf{w}(\mathbf{x}, t)=4.2857\left(2 y-y^{2}\right)$ [26]. The initial condition is a constant, i.e. $T(\mathbf{x}, 0)=15^{\circ} \mathrm{C}, \mathbf{x} \in \Omega$. The known Dirichlet boundary conditions are chosen as follows. The inflow temperature has a linear profile in $y$ and drops from $15^{\circ} \mathrm{C}$ to $0^{\circ} \mathrm{C}$ along the $y$ axis over time, i.e.

$$
T_{i n}(\mathbf{x}, t)=-30 y t+15, \quad(\mathbf{x}, t) \in \Gamma_{i n} \times\left[t_{0}, t_{f}\right]
$$

The wall temperature has a nonlinear profile in $x$ and increases from $15^{\circ} \mathrm{C}$ to $65^{\circ} \mathrm{C}$ along the $x$ axis over time, i.e.

$$
T_{\text {wall }}(\mathbf{x}, t)=100\left(1-\cos \left(\frac{\pi}{2} x\right)\right) t+15, \quad(\mathbf{x}, t) \in \Gamma_{\text {wall }} \times\left[t_{0}, t_{f}\right] .
$$

At the Neumann boundaries $\Gamma_{\text {out }}$ and $\Gamma_{r}$ a zero diffusive flux condition is used, i.e.

$$
\frac{\partial T}{\partial n}(\mathbf{x}, t)=0, \quad(\mathbf{x}, t) \in\left(\Gamma_{\text {out }} \cup \Gamma_{r}\right) \times\left[t_{0}, t_{f}\right] .
$$

The effective thermal diffusivity $a_{\text {eff }}$ is chosen to have a sinusoidal pattern over the space coordinate in the flow direction of the falling film (i.e. the $x$-direction). The wavy pattern is assumed to be time-dependent, such that the waves travel along the $x$-direction starting from a constant value at the inflow boundary $\Gamma_{\text {in }}$ (i.e. $x=$ $0 \mathrm{~mm}$ ). They propagate along the $y$ - and $z$-directions with a larger gradient in the $y$-direction (film thickness) starting from a constant value at the wall boundary $\Gamma_{\text {wall }}$ (i.e. $y=0 \mathrm{~mm}$ ), and with a comparably low gradient in the $z$-direction:

$$
\begin{aligned}
& a_{\mathrm{eff}}(\mathbf{x}, t)=a_{\mathrm{mol}}+a_{w}(\mathbf{x}, t), \\
& a_{w}(\mathbf{x}, t)=5\left(1.1+\frac{y}{5}\left(\sin \left(\pi x+\frac{t}{50}\right)+x+\frac{x z}{10}\right)\right), \\
&(x, y, z, t) \in \Omega \times\left[t_{0}, t_{f}\right] .
\end{aligned}
$$

The material properties of the fluid are lumped in the known constant molecular thermal diffusivity $a_{\mathrm{mol}}=0.35 \frac{\mathrm{mm}^{2}}{\mathrm{~s}}$, whereas the remaining part of the effective thermal diffusivity $a_{\mathrm{eff}}(\mathbf{x}, t)$ represents the unknown wavy thermal diffusivity $a_{w}(\mathbf{x}, t)$, the transport coefficient capturing the wave-induced effects in the flat film model.

In this setting, a model $f_{\mathrm{w}}(\mathbf{x}, t, \theta)$ (cf. (2.4)) for the "true" wavy thermal diffusivity $a_{w}(\mathbf{x}, t)$ in $(4.2 \mathrm{~b})$ can be formulated as

$$
\begin{array}{r}
f_{\mathrm{w}}(\mathbf{x}, t, \theta)=5\left(\vartheta_{1}+\vartheta_{2} y\left(\sin \left(\vartheta_{3} x+\vartheta_{4} t\right)+0.2 x+\vartheta_{5} x z\right)\right), \\
(x, y, z, t) \in \Omega \times\left[t_{0}, t_{f}\right], \theta \in \mathbb{R}^{5},
\end{array}
$$

where the vector $\theta=\left(\vartheta_{1}, \ldots, \vartheta_{5}\right)$ represents the vector of model parameters. A comparison of (4.2b) and (4.3) reveals the true underlying value $\theta^{e x}$ to be

$$
\theta^{e x}=(1.1,0.2, \pi, 0.02,0.1)
$$


REMARK 1. For numerical validation of the incremental identification method we postulated a model of the form (4.3) to generate temperature simulation data. In such a setting a simultaneous approach can be applied, in which the unknown parameter vector $\theta \in \mathbb{R}^{5}$ is estimated using the model in (2.12) and temperature data $T_{m}(\mathbf{x}, t)$ for the function $u(\mathbf{x}, t)$ therein. This, however, results in an optimization problem of huge complexity. We do not know of any literature in which the simultaneous approach has been applied successfully to this type of $3 D$ inverse problems. Furthermore, we emphasize, that in a practical setting the model structure is mostly not known and thus a simultaneous approach is not applicable. The first two steps of the incremental method, however, can still be applied. In the third step suitable model candidate can be identified. Due to these difficulties related to the simultaneous approach a fair comparison between the incremental and the simultaneous approach is a difficult issue and will not be treated in this paper.

In order to generate high quality temperature simulation data the nonlinear direct problem (2.12) with the "true" effective thermal diffusivity given in (4.2) is solved on a uniform fine grid with the spatial discretization consisting of $48 \times 48 \times 38$ intervals in $x, y$ and $z$ directions, respectively. This yields a space discretization with 89856 unknowns and 525312 tetrahedra.

For the solution of the inverse problems in the first two steps as well as for the solution of the parameter estimation problem in the third step of the incremental identification, we use the temperature data $T_{m}$ on the coarser grid of resolution $24 \times$ $24 \times 19$ intervals in $x, y$ and $z$ directions, respectively, to avoid so-called "inverse crime" $[21]$.

In the first step of the incremental identification procedure, we use the implicit Euler scheme with time step $\tau=0.01 \mathrm{~s}$ and apply 50 time steps starting from the initial time $t_{0}=0 \mathrm{~s}$ (i.e. $t_{f}=0.5 \mathrm{~s}$ ). For the initial approximation in the optimization procedure, due to the lack of better information, we choose $F_{w}^{0}(\mathbf{x}, t)=0,(\mathbf{x}, t) \in$ $\Omega \times[0 s, 0.5 s]$.

In the second step of the incremental identification procedure, the same boundary conditions (4.1) are used. The time interval $[0 s, 0.5 s]$ is subdivided in 50 time steps and the estimation of the wavy thermal diffusivity is carried out separately for each point in time. The initial time $t_{0}=0 \mathrm{~s}$ is a singular point, because the initial temperature is constant and no reconstruction is possible, since the coefficient is not uniquely defined in this case (cf. (2.9)).

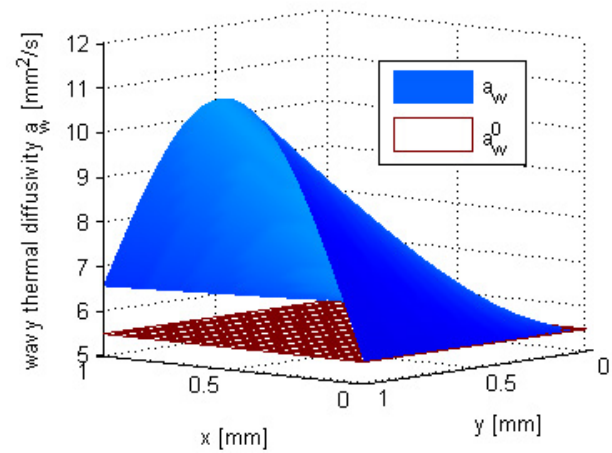

FIG. 1. Initial approximation for the wavy thermal diffusivity $a_{w}^{t, 0}$ at $t=0.01$ s and constant $z=0.5 \mathrm{~mm}$. 

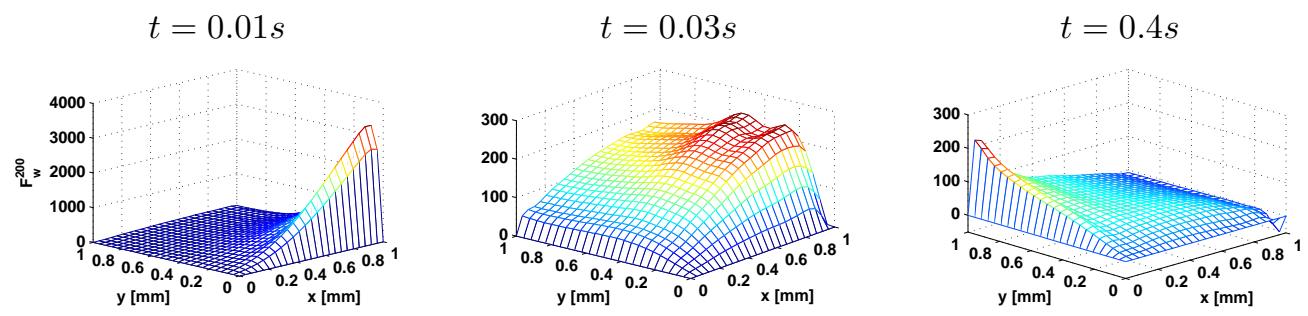

FIG. 2. Estimated source $F_{w}$ at different times for constant $z=0.5 \mathrm{~mm}$ with unperturbed measurements for nopt $=200$.

As expected, the choice of a suitable initial vector for the optimization method is much more important for the nonlinear optimization problem in the second identification step than for the linear one in the first step. In our experiment, we use the constant

$$
a_{w}^{t, 0}=5.5
$$

as initial guess for the wavy thermal diffusivity in the first time step $t=0.01 \mathrm{~s}$ (cf. Fig. 1 ). This initial guess is very different from the true solution, but coincides with the inflow $(x=0 \mathrm{~mm})$ and wall $(y=0 \mathrm{~mm})$ boundary conditions $\Gamma_{D}$. According to its boundary conditions (3.11b), the solution of the adjoint problem (3.11) is always zero along these boundaries. As a consequence, no improvement can be gained during the iteration process of the truncated Newton-CGNE method, since the search direction within the CG (inner) iteration will not be updated [2, 17]. Subsequently, neither an update is possible in the Newton (outer) iteration by (3.10). Hence, with such a choice of the initial guess we exclude the boundaries from the estimation where no information can be gained.

In the third step, the estimated functions $a_{w}^{t}(\mathbf{x})$ at time instants $t \in[0.01 s, 0.5 s]$, are used as model-based measurement data to estimate the model parameters $\theta \in \mathbb{R}^{5}$ of the model (4.3) by solving the standard least-squares problem (3.3). The initial guess $\theta^{0}=(0.5,0.5,0.5,0.5,0.5)$ is chosen in all of our computations.

Estimation results with error-free measurements will be considered first. Subsequently the estimation with artificially perturbed measurements will be analysed.

4.1. Estimation with error-free measurements. The snapshots of the source estimates $F_{w}^{n o p t}(\mathbf{x}, t)$ in the first step of the incremental identification procedure are presented in Fig. 2. In our observation, the convergence in the result was achieved after nopt $=200$ optimization iterations. During the optimization the initial approximation at the boundaries $\Gamma_{\text {in }}(x=0 \mathrm{~mm})$ and $\Gamma_{\text {wall }}(y=0 \mathrm{~mm})$ could not be improved, since the optimality condition $\nabla J_{1}\left(F_{w}\right)=0$ is achieved along these boundaries. This follows directly from the boundary conditions $(3.7 \mathrm{~b})$ of the corresponding adjoint problem (3.7) and the expression (3.6).

The estimation of the wavy thermal diffusivities $a_{w}^{t}(\mathbf{x})$ at times $t \in[0.01 s, 0.5 s]$ in the second step of the incremental approach, uses the estimated source $F_{w}^{t, 200}(\mathbf{x})$ at iteration nopt $=200$ and the temperature $T_{m}^{t}(\mathbf{x})$ at given time $t$. We choose (4.5) as the initial approximation at time $t=0.01 \mathrm{~s}$. The subdivision in time gives a flexibility to improve this initial approximation at later times. Therefore, we use already computed estimates at time instants $t$ as initial guesses for the next times $(t+\tau)$. Note, that the initial value for time $t=0.01 \mathrm{~s}$ restores the information lost at 
$t=0.01 s$
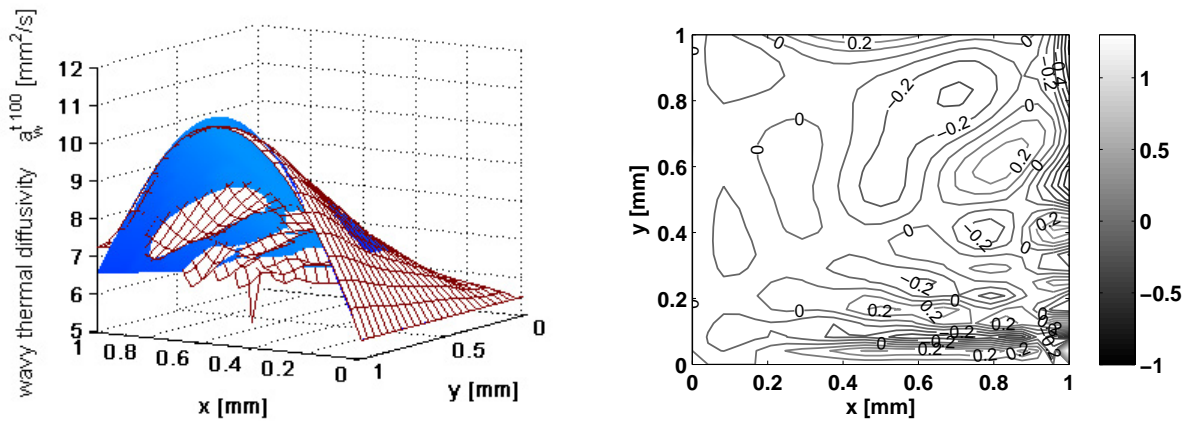

$t=0.4 s$

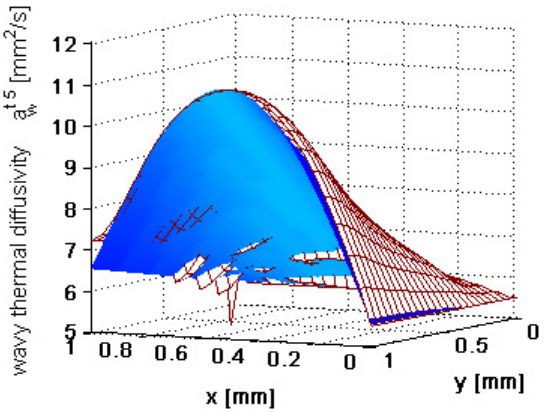

(a)

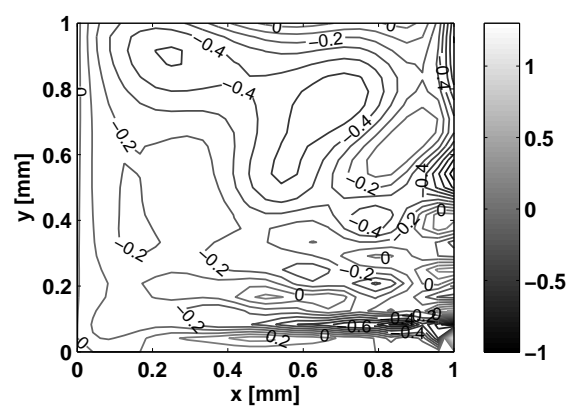

(b)

FIG. 3. (a) Estimated (meshed surface) and exact (shaded surface) wavy thermal diffusivities $a_{w}^{t}$, (b) deviation between exact and estimated wavy thermal diffusivities $a_{w}^{t}$ at different times.

the inflow and wall boundaries at which the source $F_{w}(\mathbf{x}, t)$ could not be reconstructed in the first step.

The estimates of the wavy thermal diffusivity for $z=0.5 \mathrm{~mm}$ at selected times are shown in Fig. 3 (a), whereas Fig. 3 (b) shows contour lines of the differences between the exact and estimated quantities. The estimates in these figures have been obtained as follows. At time $t=0.01 \mathrm{~s}, 100$ Newton iterations were applied. The analysis of the results for later times $t>0.01 \mathrm{~s}$ shows, that the solution converges already in

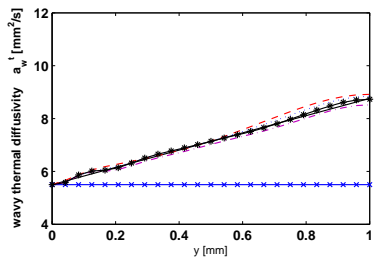

$x=0.2 \mathrm{~mm}$

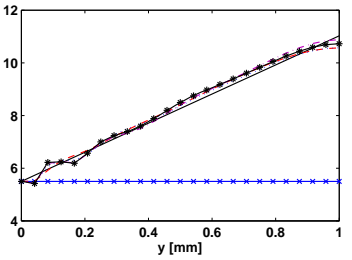

$x=0.5 \mathrm{~mm}$

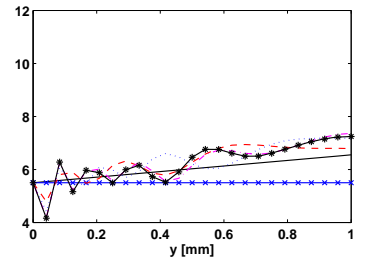

$x=1 \mathrm{~mm}$

estimated $($ nopt $=50) \cdot-\cdot-\cdot$ estimated $($ nopt $=90)-$ estimated $($ nopt $=100)$

FIG. 4. Estimated wavy thermal diffusivity $a_{w}^{t}$ at different Newton iterations nopt, time $t=$ $0.01 \mathrm{~s}$, different $x$ and constant $z=0.5 \mathrm{~mm}$. 

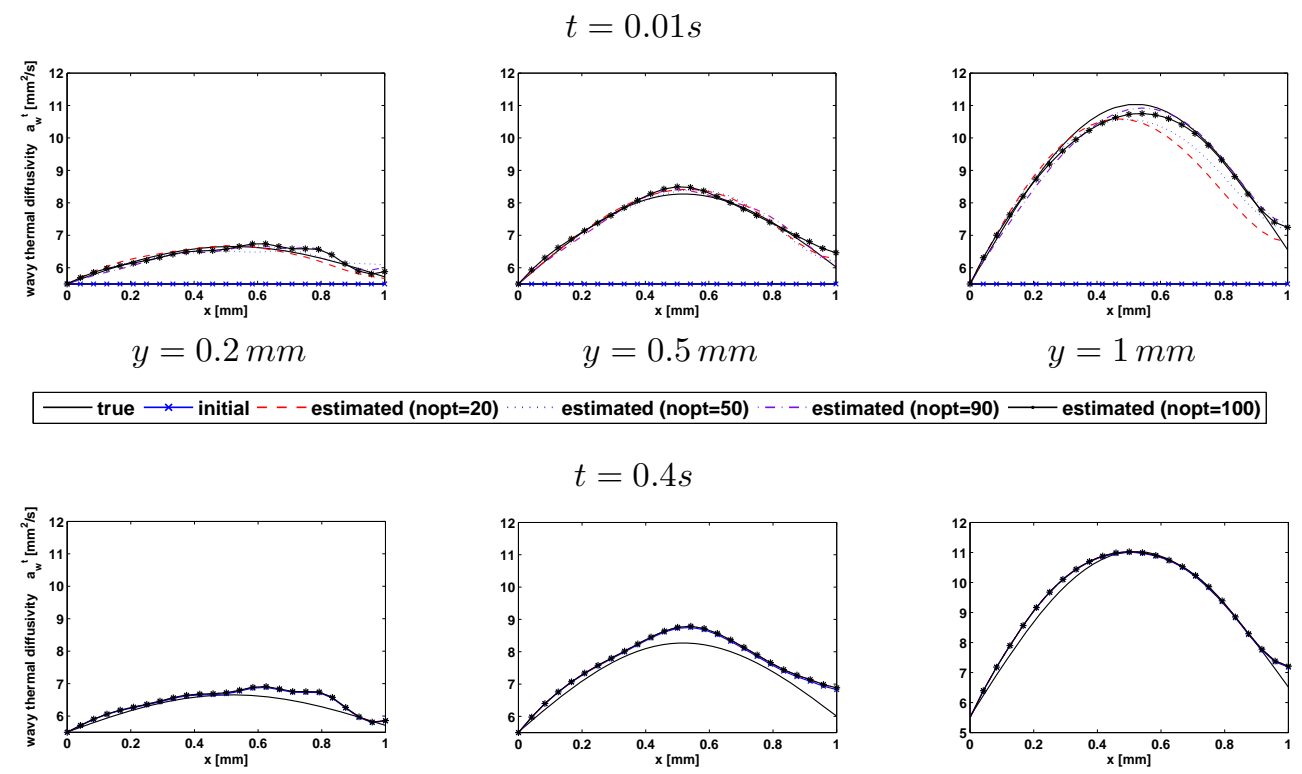

$t=0.4 s$

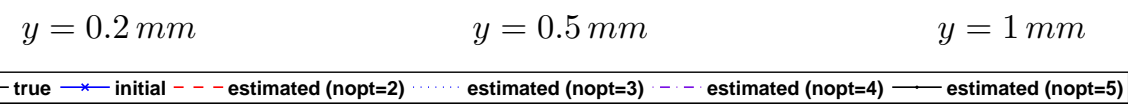

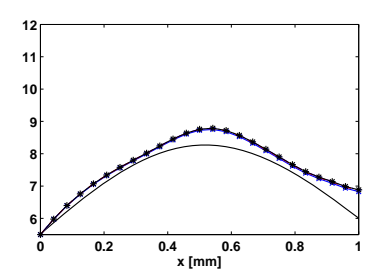

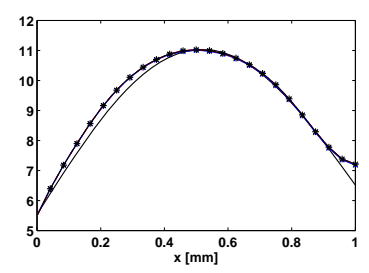

FIG. 5. Estimated wavy thermal diffusivity $a_{w}^{t}$ at different Newton iterations nopt, two different times, three different $y$ and constant $z=0.5 \mathrm{~mm}$.

5 Newton iterations. Moreover, it is possible to reduce the number of the inner iterations remarkably, because very good initial values are available at later times. A closer look at the results reveals that, independent of time, the estimation quality decreases in the $x$-direction (i.e. the direction of flow) by approaching the outflow boundary $\Gamma_{\text {out }}$ (i.e. $x=1 \mathrm{~mm}$ ). This can be observed in Fig. 4, where the estimates are presented exemplarily for $t=0.01 \mathrm{~s}$ as a functions of $y$ for $x \in\{0.2,0.5,1\} \mathrm{mm}$ and $z=0.5 \mathrm{~mm}$. The development of the estimates for different numbers of Newton iterations nopt is shown in the figures, too. The reason of this distortion is, that at the outflow boundary $\Gamma_{\text {out }}$ the estimation quality of the source $F_{w}$ in the previous step is impaired by the lack of information as at this boundary; due to convection, not enough information is available for the reconstruction of the unknown founction from the data.

The estimates of the wavy thermal diffusivity at different numbers of Newton iterations nopt as a functions of $x$ for $y \in\{0.2,0.5,1\} \mathrm{mm}$ and $z=0.5 \mathrm{~mm}$ at times $t=$ $\{0.01,0.4\} s$ are presented in Fig. 5. Because of the very good initial approximation, at large times (e.g. for $t=0.4 \mathrm{~s}$ in the Figure) the convergence is achieved even before reaching nopt $=5$.

In the third identification step the model parameters $\theta \in \mathbb{R}^{5}$ are estimated using the reconstructed transport coefficients $a_{w}^{t}(\mathbf{x})$ at times $t \in[0.01 s, 0.5 s]$ and the proposed model $f_{\mathrm{w}}(\mathbf{x}, t, \theta)$ (cf. (4.3)). Fig. 6 (a) shows the deviations between the reconstructed wavy thermal diffusivities $a_{w}^{t}(\mathbf{x})$ and the optimal solution $f_{\mathrm{w}}\left(\mathbf{x}, t, \theta^{\star}\right)$ for selected times $t \in\{0.01,0.4\} \mathrm{s}$. A high reconstruction quality is achieved. The 


$$
t=0.01 s
$$
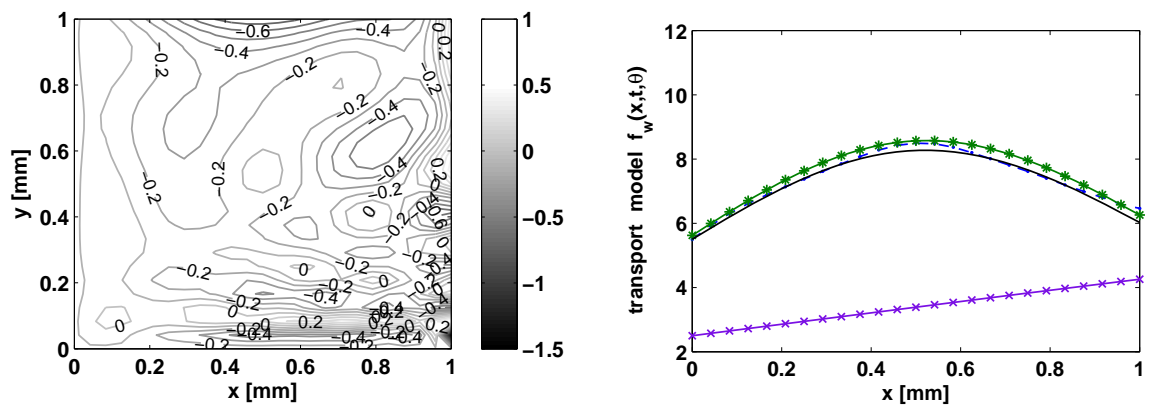

$t=0.4 s$

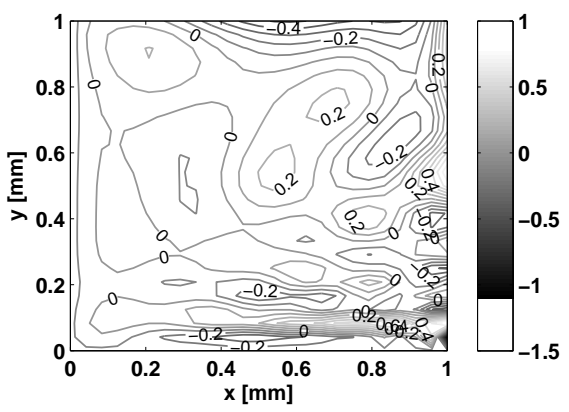

(a)

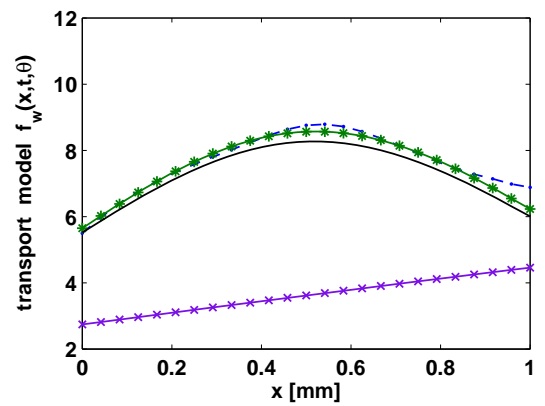

(b)

* initial - - - estimation (step II) —* estimation (step III) — true

FIG. 6. (a) Deviation between the wavy thermal diffusivity estimated in the second step and the model $f_{w}$ estimated in the third step of the incremental approach (b) "true" wavy thermal diffusivity, estimated wavy thermal diffusivity $a_{w}^{t}$ in the second step and estimated model $f_{w}$ in the third step of the incremental approach at different times for constant $y=0.5 \mathrm{~mm}$ and $z=0.5 \mathrm{~mm}$

resulting optimal value for the model parameter vector

$$
\theta^{\star}=(1.12,1.05,3.12,0.02,0.06),
$$

is in a good agreement with the exact value $\theta^{e x}$ in (4.4). In Fig. 6 (b) the estimation results for constant $y=0.5 \mathrm{~mm}$ and $z=0.5 \mathrm{~mm}$ at different identification steps of an incremental approach are presented together with the "true" wavy thermal diffusivity $a_{w}^{t}(\mathbf{x})$ (cf. (4.2b)) and their initial guesses for a detailed comparison. There is an obvious bias which can be atributed to error propagation in the incremental identification procedure [5]. This bias can be easily eliminated by a final simultaneous step which converges quickly due to very good initial values [9].

4.2. Estimation in the presence of measurement errors. In this section, we perturb the measured temperature $T_{m}$ by an artificial measurement error $\omega$. The values of $\omega$ are generated from a zero mean normal distribution with variance one. We compute the perturbed temperature $\tilde{T}_{m}$ by

$$
\tilde{T}_{m}=T_{m}+\sigma \omega,
$$

with $\sigma$ being the standard deviation of the measurement error. The parameter $\sigma$ is used to control the amount of error added to the exact data. We take the value 


$$
t=0.01 s
$$

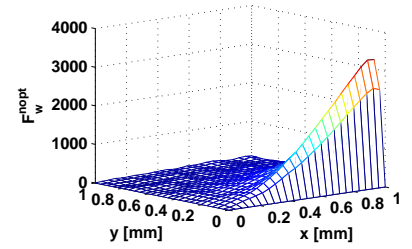

$t=0.03 s$

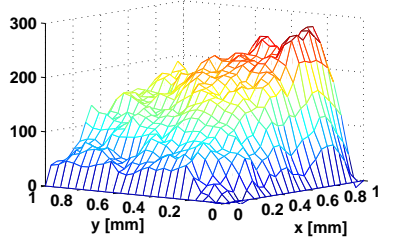

$t=0.4 s$

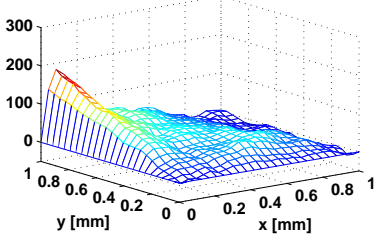

FIG. 7. Estimated source $F_{w}$ at different times for constant $z=0.5 \mathrm{~mm}$ with perturbed measurements $(\sigma=0.1)$ for nopt $=100$.

$\sigma=0.1$ in the following simulation experiments.

In the presence of measurement errors, an increasing number of iterations eventually leads to a poorer estimation quality due to the undesirable effect that measurement errors are resolved. Therefore, a compromise between the residual and the solution norm has to be established by an appropriate regularization [19]. Besides the (fixed) regularizing effect of time and space discretization, the number of optimization iterations is used as a regularization parameter. An appropriate value for this parameter can be obtained by the L-curve, which is a parameterized plot of the residual against a smoothing norm of the solution.

The results of source term estimation will be presented first. The L-curve method suggested nopt $=100$ as a reasonable choice of the regularization parameter for the given value of $\sigma$. The snapshots of the regularized optimal estimates $F_{w}^{n o p t}(\mathbf{x}, t)$ are shown in Fig. 7, for constant $z=0.5 \mathrm{~mm}$. Due to the errors in the measurements, the estimates are no longer smooth, but the qualitative behaviour is the same as for the case without measurement noise (cf. Fig. 2). This can be in detail observed in Fig. 8, where the results of the estimation $F_{w}^{\text {nopt }}(\mathbf{x}, t)$ obtained with perturbed data is compared to the estimates $F_{w}^{200}(\mathbf{x}, t)$ obtained with exact data in the previous section.

The regularization parameter can be determined alternatively using the discrepancy principle [14]. Here the knowledge of the error's magnitude is used to propose the stopping condition for the objective functional: the iterationsis stopped when the residual approximately equals the error level $\sigma$. Using (3.1), we get the condition

$$
J_{1}\left(F_{w}^{n}\right)<\kappa_{1}\left(t_{f}-t_{0}\right) V \sigma,
$$

where $V$ is the volume of $\Omega$ and $\kappa_{1}>1$. For a value of $\kappa_{1}=1.01$ the optimal number
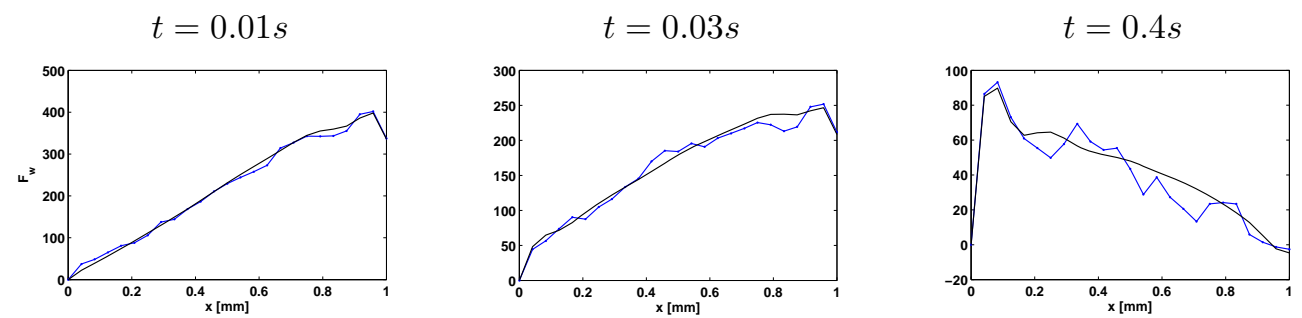

estimation with $\mathrm{T}_{\mathrm{m}}+\sigma \omega \longleftarrow$ estimation with $\mathrm{T}_{\mathrm{m}}$

FIG. 8. Estimated source $F_{w}$ with unperturbed measurements for nopt $=200$ and perturbed measurements for $\sigma=0.1$ for (nopt $=100$ ) at different times. 


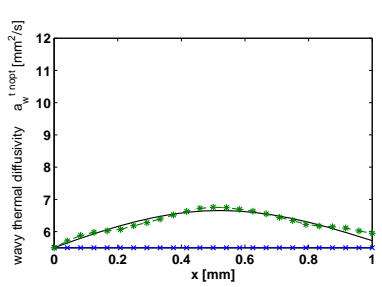

$t=0.01 s$
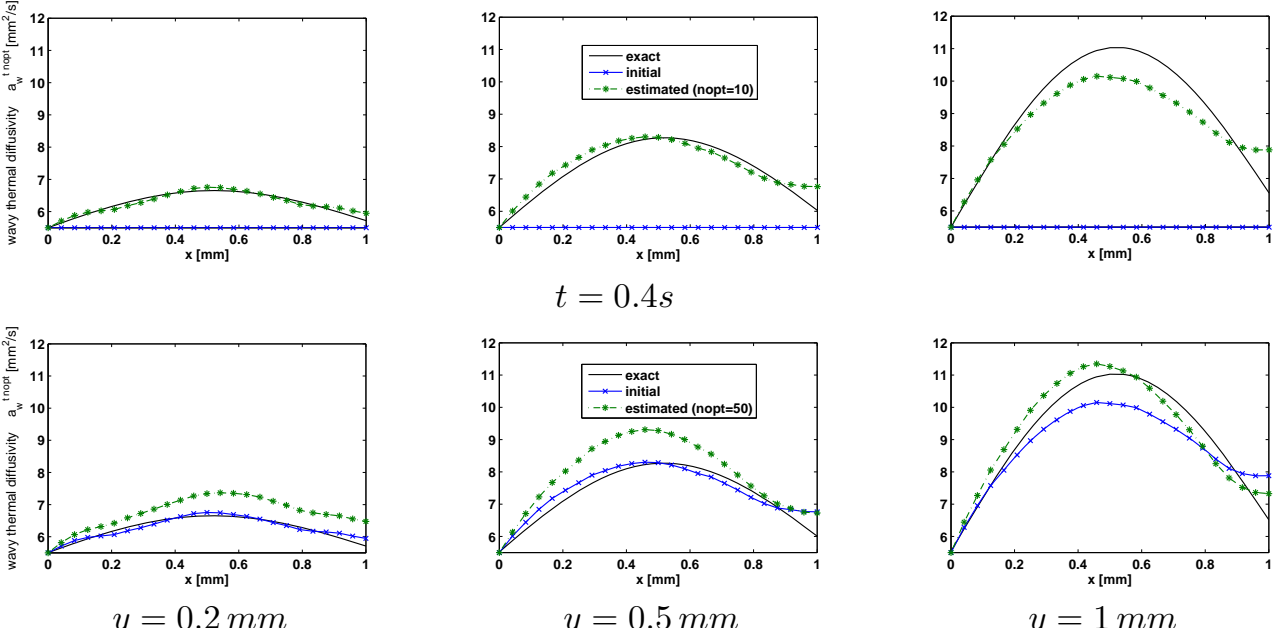

$t=0.4 s$

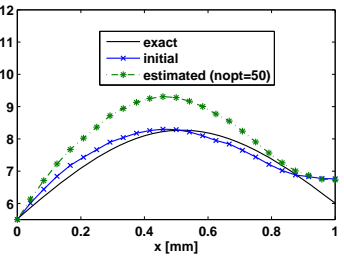

$y=0.5 \mathrm{~mm}$

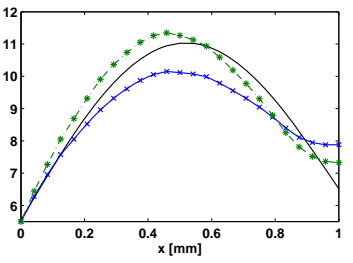

$y=1 \mathrm{~mm}$

FIG. 9. Estimated wavy thermal diffusivity $a_{w}^{t}$ for noise level of $\sigma=0.1$ after corresponding optimal nopt iterations at different times for constant $z=0.5 \mathrm{~mm}$ and different $y$.

of iterations is nopt $=16$ for a given error $\sigma=0.1$. The estimate obtained at this point is smoother, however over-regularized, whereas the one suggested by the L-curve method shows oscillations but is closer to the estimations obtained with unperturbed data. Therefore, we took the estimates obtained by the L-curve principle in the next identification step.

For the estimation of the wavy thermal diffusivities $a_{w}^{t}(\mathbf{x})$ at times $t \in[0.01 s, 0.5 s]$, the regularized optimal solution $F_{w}^{t, n o p t}(\mathbf{x})$ and the measurement data $\tilde{T}_{m}^{t}$ are used in the corresponding direct problem (2.10) for a given time $t$. We apply the L-curve and the discrepancy principle in order to find the optimal value for the number of Newton iterations in the truncated Newton-CGNE method. The stopping condition for the Newton iteration based on the discrepancy principle is

$$
J_{2}\left(a_{w}^{t, k}\right)<\kappa_{2} V \sigma .
$$

In experiments we tried different values of $\kappa_{2}>1$ and always observed that the estimates obtained by the discrepancy principle are smoother but still over-regularized, whereas those suggested by the L-curve method contain oscillations but are closer to the exact quantity. The same result has been observed for source estimation in the first step for the example considered. A good understanding of the regularizing effects of the CG method is well developed for linear problems [19], whereas a practical understanding of the regularizing effect for the truncated Newton-CGNE method is rather speculative [18]. Nevertheless, the choice of an appropriate value for the regularization parameter is rather problem-dependent and relies to a large extent on user experience. In Fig. 9, the optimal regularized estimates for the chosen noise level $\sigma=0.1$ obtained from the L-curve method are presented as a function of $x$ for $z=0.5 \mathrm{~mm}$ and different values of $y$ at selected times. Due to the reasons stated above in the noise-free case, we see again, that estimation quality decreases near the outflow at $x=1 \mathrm{~mm}$. Here, in contrast to the noise-free case, the estimates computed for $t=0.01$ have been used as initial approximations for each later time $t>0.01 \mathrm{~s}$. 


$$
t=0.01 \mathrm{~s}
$$
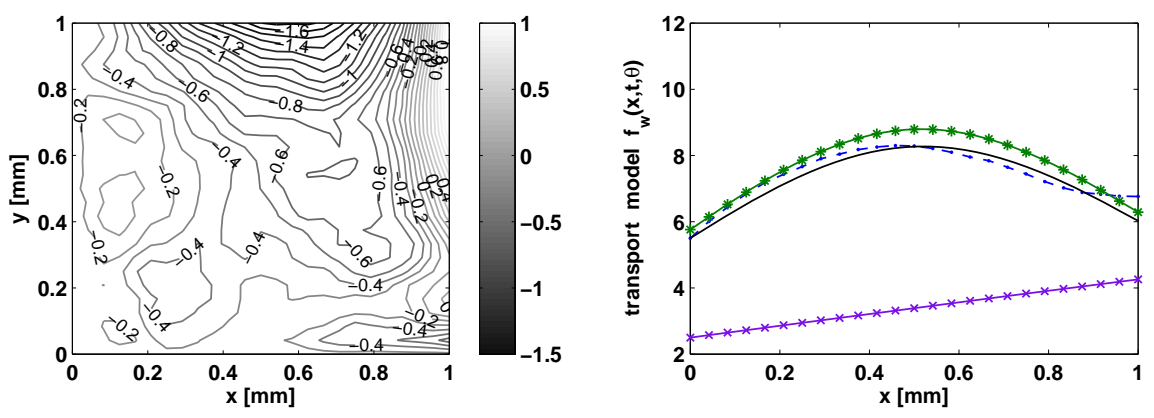

$t=0.4 s$

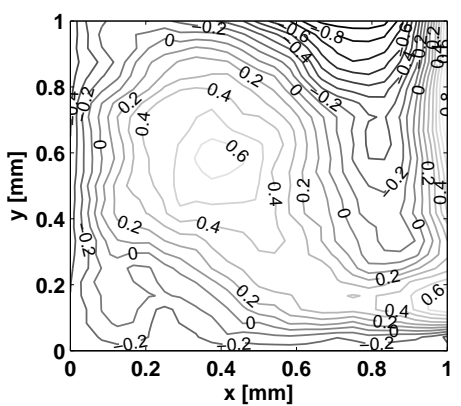

(a)
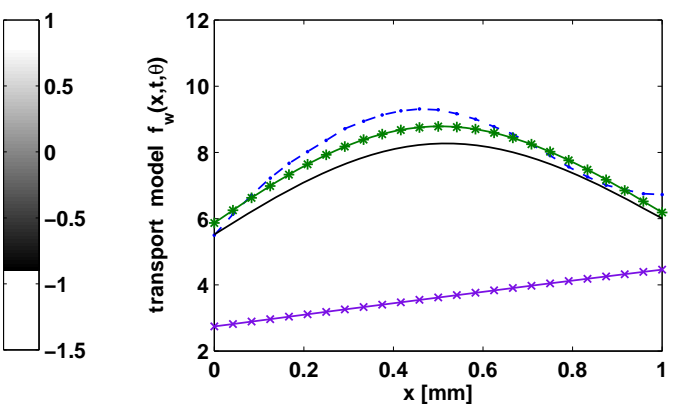

(b)

$\longrightarrow$ initial --- regularized estimation (step II) $\longrightarrow$ * estimation (step III) - true

FIG. 10. (a) Deviation between the regularized wavy thermal diffusivity estimated in the second step and the model $f_{w}$ estimated in the third step of the incremental approach at different times for the noise-level $\sigma=0.1$. (b) "true" wavy thermal diffusivity, regularized wavy thermal diffusivity $a_{w}^{t \text { nopt }}$ in the second step and estimated model $f_{w}$ in the third step of the incremental approach at different times for constant $y=0.5 \mathrm{~mm}$ and $z=0.5 \mathrm{~mm}$ for the noise-level $\sigma=0.1$.

Finally, in order to estimate the model parameters $\theta \in \mathbb{R}^{5}$ in the model $f_{\mathrm{w}}(\mathbf{x}, t, \theta)$ (cf. (4.3)) the regularized optimal solutions $a_{w}^{t}{ }^{\text {nopt }}(\mathbf{x})$ at times $t \in[0.01 s, 0.5 s]$ are used. In Fig. 10 (a) the deviations between the optimal regularized wavy thermal diffusivity from the second step and the optimal, estimated model $f_{\mathrm{w}}\left(\mathbf{x}, t, \theta^{\star}\right)$ in the third step are presented for selected times. The resulted optimal value of the parameter vector amounts to

$$
\theta^{\star}=(1.15,1.08,3.17,0.09,0.08)
$$

In Fig. 10 (b) the estimations at different identification steps are presented once more together with the "true" wavy thermal diffusivity $a_{w}^{t}(\mathbf{x})$ (cf. (4.2a)) for constant $y=0.5 \mathrm{~mm}$ and $z=0.5 \mathrm{~mm}$ and selected times. The estimation quality has decreased compared to the noise-free case above, however, a quite good reconstruction has been achieved.

5. Conclusions. A novel method for the incremental identification of transport models for transport coefficients in convection-diffusion systems is presented. The simultaneous model is split into three hierarchically structured submodels. The iden- 
tification problems in the first two steps (levels) have to be solved only once. The model for the transport coefficient has to be estimated in the third step.

The approach is illustrated for the identification of a model for an effective thermal diffusivity in a three-dimensional convection-diffusion problem which is similar to a flat film model used to investigate energy transport in laminar wavy film flows. The first step of the incremental identification is rather easy to handle due to the linearity of the corresponding source inverse problem. The results obtained with a CG method at this level are quite satisfactory both for error-free and noisy measurements. The second step of the identification is far more complex due to the strong nonlinearity and high degree of ill-posedness of the coefficient inverse problem that has to be solved. The truncated Newton-CGNE method, belonging to the class of inexact Newtontype methods, is used to solve this problem as it is known to be very suitable for such nonlinear inverse problems [14]. For good results one needs, however, an initial approximation which is sufficiently close to the solution. Finally, in the third step, a single model for the effective thermal diffusivity is considered and the parameter estimation for it is carried out. The interplay between the tree steps both with and without measurement errors is investigated by means of an illustrative case study.

We have, for the first time, successfully applied the concept of incremental model identification to a complicated transport problem in 3D. This proof of concept should not address the model discrimination issue in the third step of the incremental approach, where the best model is chosen from a set of candidate models by discriminating between the candidates using some reasonable model fit criterion [29].

Future work will address the following issues in addition to model discrimination. Robust regularization techniques will be studied in more detail. Besides the number of iterations, the discretization in space and time has a regularizing effect which needs to be properly exploited in an appropriate discretization framework. Furthermore, ill-posedness can be handled by adding a (Tikhonov) regularization term to the corresponding objective functional, cf. [14]. The interplay between such a regularization on the level of the problem with those regularizing effects in the numerical method, have to be analysed carefully (see e.g. $[4,7]$ ). A further issue is a better theoretical understanding of the error propagation through the sequence of inverse problems in the incremental approach similar to [5]. Finally, in this paper we do not present a detailed comparison with the simultaneous approach (as in [5]), which is also a topic of current research.

Acknowledgements. The authors gratefully acknowledge the financial support of Deutsche Forschungsgemeinschaft (DFG) within the Collaborative Research Center (SFB) 540 "Model-based experimental analysis of kinetic phenomena in fluid multiphase reactive systems".

\section{REFERENCES}

[1] O. M. Alifanov, Inverse Heat Transfer Problems, Springer, Berlin, 1994.

[2] O. M. Alifanov, E.A. Artyukhin, and S.V. Rumyantsev, Extreme Methods for Solving IllPosed Problems with Applications to Inverse Heat Transfer Problems, Begell House, Inc., U.S.A., 1995.

[3] O. M. Alifanov, Y. Jarny, P.V. Prosuntsov, and G.A. Ivanov, Complex identification of thermophysical properties of anisotropic composite material, in Proceedings of the 5th international Conference on Inverse Problems in Engineering: Theory and Practice, Cambridge, U.K., 2005.

[4] U. Ascher And E. HABER, A multigrid method for distributed parameter estimation problems, Electronic Transactions on Numerical Analysis, 15 (2003), pp. 1-17. 
[5] A. Bardow AND W. Marquardt, Incremental and simultaneous identification of reaction kinetics: methods and comparison, Chem. Eng. Sci., 59 (2003), pp. 2673-2684.

[6] - Identification of diffusive transport by means of an incremental approach, Comp. Chem. Eng., 28 (2004), pp. 585-595.

[7] Th. Binder, L. Blank, W. Dahmen, and W. Marquardt, On the regularization of dynamic data reconciliation problems, 12 (2002), pp. 557-567.

[8] H. Brauer, Strömung und Wärmeübergang bei Rieselfilmen, in VDI-Forsch.-Heft 457, VDIVerlag, Düsseldorf, 1956.

[9] M. Brendel, D. Bonvin, and W. Marquardt, Incremental identification of kinetic models for homogeneous reaction systems, Chem. Eng. Sci., 61 (2006), pp. 5404-5420.

[10] R. S. Brodkey and H. C. Hershey, Transport Phenomena - A Unified Approach, McGrawHill Book Company, U.S.A, 1976.

[11] M. J. Colaco, G. S. Dulikravich, H. R. B. Orlande, and F. A. Rodrigues, A comparison of two solution techniques for the inverse problem of simultaneously estimating the spatial variations of diffusion coefficient and source terms, in Proceedings of IMECE'03, 2003 ASME International Mechanical Engineering Congress and Exposition, Washington, D.C., U.S.A., 2003.

[12] G. Dietze, V.V. LeL, And R. Kneer, Modelling of heat transfer in stable wavy film flow based on effective thermal diffusivity, in Proceedings of IHTC-13 2006, Sidney, 2006.

[13] DROPS package. http://www.igpm.rwth-aachen.de/DROPS/.

[14] H. W. Engl, M. Hanke, and A. Neubauer, Regularization of Inverse Problems, Kluwer Academic Publishers, Dordrecht, 1996.

[15] R. Fletcher and C. M. Reeves, Function minimization by conjugate gradients, J. Comput., 7 (1964), pp. 149-154.

[16] A. L. Frenkel and K. Indireshkumar, Wavy film flows down an inclined plane: Perturbation theory and general evolution equation for the film thickness, Phys. Rev. E, 60 (1999), pp. 4143-4157.

[17] S. Gross, M. Soemers, A. Mhamdi, F. Al Sibai, A. Reusken, W. Marquardt, and U. Renz, Identification of boundary heat fluxes in a falling film experiment using high resolution temperature measurements, Int. J. Heat Mass Tran., 48 (2005), pp. 5549-5562.

[18] M. HANke, Regularizing properties of a truncated Newton-CG algorithm for nonlinear inverse problems, Numer. Funct. Anal. Optim., 18 (1998), pp. 971-993.

[19] C. Hansen, Rank-Deficient and Discrete Ill-posed Problems: Numerical Aspects of Linear Inversion, SIAM Monographs on Mathematical Modeling and Computation, SIAM, Philadelphia, 1998.

[20] T. Hastie, R. Tibshirani, and J. Friedman, The elements of statistical learning: Data mining, Inference, and Prediction, Springer Verlag, New York, 2001.

[21] J. P. Kaipio and E. Somersalo, Computational and Statistical Methods for Inverse Problems, Springer Verlag, New York, 2004.

[22] W. MARquARDt, Towards a process modeling methodology, vol. 293 of NATO-ASI Series, Kluwer Academic Pub., Dordrecht, 1995, pp. 3-41.

[23] — Model-based experimental analysis of kinetic phenomena in multi-phase reactive systems, Chem. Eng. Res. Des., 83 (2005), pp. 561-573.

[24] J. Nocedal and S. J. Wright, Numerical Optimization, Springer Series in Operations Research, Springer, Berlin, Heidelberg, New York, 1999.

[25] M.K. Panga, R. R. Mudunuri, and V. Balakotaiah, Long-wavelength equation for vertically falling films, Phys. Rev. E, 71 (2005), p. 036310.

[26] L. PrandtL, Über Flüssigkeitsbewegung bei sehr kleiner Reibung, in Gesammelte Abhandlungen zur angewandten Mechanik, Hydro- und Aerodynamik, W. Tolmien, H. Schlichting, and H. Görtler, eds., vol. 2, Springer-Verlag, Berlin, 1961.

[27] Y. SAAD, Iterative Methods for Sparse Linear Systems, SIAM, Philadelphia, PA, USA, 2003.

[28] J. Su AND F. H. GEOFFREY, Inverse heat conduction problem of estimating time-varying heat transfer coefficient, Numer. Heat Transf., 45 (2004), pp. 777-789.

[29] E. Walter and L. Pronzato, Identification of Parametric Models from Experimental Data, Springer, Berlin, 1997.

[30] W. WiLKE, Wärmeübergang an Rieselfilmen, in VDI-Forsch.-Heft 490, VDI-Verlag, Düsseldorf, 1962. 